\section{Decreased Expression of Nicotinamide Phosphoribosyltransferase in Patients with Juvenile Idiopathic Arthritis Receiving Methotrexate}

To the Editor:

Nicotinamide phosphoribosyltransferase (NAMPT), also known as pre-B cell colony-enhancing factor or visfatin, is an adipocytokine that has been identified as a proinflammatory mediator in cardiovascular disease, pulmonary inflammation, and sepsis ${ }^{1}$. Several roles have been ascribed to NAMPT including possible insulin mimetic properties, a part in the nicotinamide adenine dinucleotide (NAD) salvage pathway, and cytokine induction $^{2,3,4,5}$. Recently, its effects have been studied in rheumatoid arthritis (RA), systemic lupus erythematosus, and systemic sclerosis ${ }^{6,7}$. NAMPT has been shown to be produced by synovial tissue, subintima, lymphocytes, and vascular endothelial cells ${ }^{7}$. In RA, it has been shown to activate human leukocytes, induce proinflammatory cytokines including interleukin $1 \beta$ (IL-1 $\beta$ ), IL-6, and tumor necrosis factor- $\alpha$ (TNF- $\alpha$ ), and protect fibroblasts and neutrophils from apoptosis, perpetuating inflammation and resulting in joint destruction ${ }^{7,8,9}$. NAMPT concentrations have also been shown to correlate with $\mathrm{C}$-reactive protein levels and clinical disease activity in patients with $\mathrm{RA}^{8}$.

APO866 is a molecule in development that inhibits the enzymatic function of NAMPT, leading to significantly lower levels of intracellular NAD, IL-1 $\beta$, IL-6, and TNF- $\alpha$ in mice with collagen-induced arthritis ${ }^{10}$. This has garnered attention in studies of adults with rheumatic diseases but little research has been conducted in the pediatric population, particularly children with juvenile idiopathic arthritis (JIA). To determine if NAMPT inhibitors have the potential to affect pediatric disease, we tested the hypotheses that NAMPT concentrations are (1) increased in subjects with active arthritis, (2) decreased in patients treated with methotrexate (MTX), and (3) decreased by anti-TNF- $\alpha$ therapy in a cross-sectional cohort of patients with JIA.

In a single-center cross-sectional study, plasma samples from 115 patients with JIA receiving stable doses of MTX and 80 patients not receiving MTX were combined and analyzed for NAMPT concentrations. Clinical data were collected by chart review and included age, diagnosis, presence of active arthritis, number of active joints, body mass index (BMI), erythrocyte sedimentation rate (ESR), MTX use, MTX dose in $\mathrm{mg} / \mathrm{kg}$, anti-TNF- $\alpha$ use, and alternative disease-modifying antirheumatic drug (DMARD) and/or corticosteroid use. Plasma concentrations of NAMPT were measured using a human NAMPT-specific carboxy-terminal enzyme immunoassay kit. Two single-nucleotide polymorphisms (SNP) in the NAMPT promoter region $(-1001 \mathrm{~T}>\mathrm{G}$ and $-1535 \mathrm{C}>\mathrm{T})$ were genotyped using a 5'-nuclease-based assay. NAMPT concentrations were log-transformed and univariate statistical analyses were performed using chi-square tests, $\mathrm{t}$ tests, and ANOVA as appropriate. Log-transformed values were back-transformed to geometric mean values. Variables found to be significant in univariate analyses $(\mathrm{p}<0.05)$ and variables deemed to be clinically relevant were entered into a multivariate linear regression model.

A total of 195 patients with JIA were included in the study. Median (interquartile range) age of the population was 133 months $(78,169)$ and the median NAMPT concentration was $19.7 \mathrm{ng} / \mathrm{ml}(15.3,25.8)$. Characteristics of patients are summarized in Table 1. In univariate analyses of the entire cohort, NAMPT concentrations were increased in subjects demonstrating active arthritis (mean $20.5 \pm 1.4 \mathrm{vs} 18.6 \pm 1.4 \mathrm{ng} / \mathrm{ml}$; $\mathrm{p}=0.05)$. Patients receiving MTX had lower concentrations of NAMPT than those not receiving the drug $(18.6 \pm 1.4 \mathrm{vs} 21.4 \pm 1.4 \mathrm{ng} / \mathrm{ml} ; \mathrm{p}=0.02)$, but anti-TNF- $\alpha$ therapy had no effect $(19.9 \pm 1.5 \mathrm{vs} 19.6 \pm 1.4 \mathrm{ng} / \mathrm{ml} ; \mathrm{p}=$ $0.8)$. There were no associations between NAMPT concentrations and age, JIA subtype (including specifically systemic JIA), number of active joints, MTX dose, BMI, or ESR. There were also no differences in NAMPT concentrations compared with the presence of NAMPT promoter region SNP. In a multivariate linear regression model controlling for MTX use, anti-TNF- $\alpha$ use, active arthritis, and number of active joints, the use of
Table 1. Demographics and clinical data of the cohort of patients with juvenile idiopathic arthritis (JIA; $\mathrm{n}=195$ ).

Characteristic

Age, mo, median (interquartile range)

$133(78,169)$

JIA subtype, n (\%)

Systemic

$22(11.3)$

Oligoarticular

33 (16.9)

Oligoarticular extended

RF-negative polyarticular

$21(10.8)$

$71(36.4)$

RF-positive polyarticular

$10(5.1)$

Enthesitis-related arthritis

29 (14.9)

Psoriatic arthritis

9 (4.6)

NAMPT concentration, median $\mathrm{ng} / \mathrm{ml}$

(interquartile range)

$19.7(15.3,25.8)$

Body mass index, median \% (interquartile range)

ESR, median $\mathrm{mm} / \mathrm{h}$ (interquartile range)

Presence of active arthritis, $\mathrm{n}(\%)$

No. active joints

$18.2(15.7,22.1)$

$11.5(8,22)$

$112(57.4)$

$1(0,4)$

$115(59)$

Methotrexate use, n (\%)

$56(28.7)$

NAMPT: Nicotinamide phosphoribosyltransferase; RF: rheumatoid factor ESR: erythrocyte sedimentation rate.

MTX [coefficient $\mathrm{B}=-0.02 \pm 0.01(95 \% \mathrm{CI}-0.05,-0.003), \mathrm{p}=0.03$ ] and presence of active arthritis [coefficient $\mathrm{B}=0.03 \pm 0.01$ (95\% CI 0.002 , $0.050), \mathrm{p}=0.04]$ remained significant predictors of NAMPT concentrations.

The cohort was further stratified based upon MTX drug use. Of the 115 subjects receiving MTX, 54 (47\%) received nonsteroidal antiinflammatory drugs (NSAID), 10 (9\%) received oral prednisone at doses $\leq 15 \mathrm{mg} /$ day, and $36(31 \%)$ received anti-TNF- $\alpha$ agents. None of the subjects in this group received alternative DMARD agents. The 80 subjects not receiving MTX had no statistically significant differences in NSAID use $(51 \%)$, oral steroid use $(6 \%)$, or anti-TNF- $\alpha$ use $(25 \%)$. There was a significant difference in the frequency of alternative DMARD use $(6 \%$ vs $0 \%$; $\mathrm{p}=$ $0.01)$. The cohorts were then further analyzed excluding all subjects that received anti-TNF- $\alpha$ agents, corticosteroids, and alternative DMARD to compare subjects on $(n=73)$ and off $(n=53)$ MTX (with the inclusion of NSAID only). When the above univariate analyses were repeated in this select cohort of patients, the difference in NAMPT concentrations, although lower, was no longer statistically significant in subjects receiving $\operatorname{MTX}(19.3 \pm 0.13$ vs $21.2 \pm 0.14 \mathrm{ng} / \mathrm{ml} ; \mathrm{p}=0.1)$. However, the difference in NAMPT concentrations in patients who had active arthritis remained significantly higher $(21.4 \pm 0.13$ vs $18.7 \pm 0.14 \mathrm{ng} / \mathrm{ml} ; \mathrm{p}=0.01)$. Again, there were no associations between NAMPT concentrations and age, JIA subtype (specifically including patients with systemic JIA), number of active joints, MTX dose, BMI, ESR, or the presence of NAMPT SNP. In the multivariate linear regression model controlling for MTX use, active arthritis, and number of active joints, only the presence of active arthritis [coefficient $\mathrm{B}=0.03 \pm 0.01(95 \% \mathrm{CI} 0.006,0.06), \mathrm{p}=0.02$ ] remained a significant predictor of NAMPT concentrations in this select cohort. Interestingly, when the group of remaining subjects receiving anti-TNF- $\alpha$ agents, corticosteroids, and/or alternative DMARD were compared, those subjects receiving MTX in addition to these other agents had significantly lower NAMPT concentrations compared to those who did not $(20.8 \pm 0.17$ vs $24.8 \pm 0.15 \mathrm{ng} / \mathrm{ml} ; \mathrm{p}=0.04)$, and subjects receiving anti-TNF- $\alpha$ agents had lower concentrations of NAMPT, but not significantly so $(21.4 \pm 0.16$ vs $26.6 \pm 0.17 \mathrm{ng} / \mathrm{ml} ; \mathrm{p}=0.06)$, suggesting a potentially synergistic effect between MTX and anti-TNF- $\alpha$ agents in lowering plasma NAMPT concentrations.

These preliminary findings in patients with JIA demonstrate that 
NAMPT is elevated in patients with active arthritis compared to those without, and decreases in response to treatment with MTX, potentially enhanced by the use of anti-TNF- $\alpha$ agents. Of note, subjects receiving MTX had significantly lower levels of NAMPT, even after controlling for active arthritis and anti-TNF- $\alpha$ use, and anti-TNF- $\alpha$ use did not appear to decrease NAMPT concentrations significantly when investigated by itself. The explanation for lower concentrations of NAMPT in subjects treated with MTX is not immediately apparent, because the complex signaling pathways between NAMPT and inflammatory cytokines are not fully elucidated. MTX is known to inhibit aminoimidazole carboxamide ribonucleotide (AICAR) transformylase, which results in accumulation of AICAR that further sensitizes AMP-activated protein kinase (AMPK) ${ }^{11}$. AMPK has been shown to induce NAMPT transcription in glucose-restricted skeletal myoblasts, which may appear to contradict our findings here; however, AICAR (through activation of AMPK) has also been shown to inhibit nuclear factor- $\kappa \mathrm{B}(\mathrm{NF}-\kappa \mathrm{B})^{12,13}$. NF- $\kappa \mathrm{B}$ has been shown to induce NAMPT activity, thus inhibition of this important proinflammatory transcription factor in MTX-treated patients may contribute to lower NAMPT concentrations in these patients. Further research is needed to unravel these complex interactions ${ }^{14}$. The apparently synergistic effect of MTX and anti-TNF- $\alpha$ agents in lowering NAMPT concentrations may also explain the improved efficacy of combination drug therapy seen clinically.

As we strive for safe and effective therapy for childhood arthritis, we are limited by factors including incomplete understanding of the etiology of JIA, few validated outcomes measures for disease activity, and a paucity of novel therapeutic targets. Our exploratory cross-sectional study had several limitations including a sample size insufficiently powered to analyze NAMPT concentrations stratified by JIA subtype and uncontrolled medication use, which allows for the potential of confounding by indication. Nevertheless, these data represent "real world" clinical patients, applicable and possibly generalizable to a large population of patients; and identify NAMPT and other adipocytokines as potential biomarkers and therapeutic targets in JIA, subject to replication and validation.

EMILY J. FOX, MD, Department of Pediatrics; J. STEVEN LEEDER, PharmD, PhD, Chief, Division of Clinical Pharmacology and Medical Toxicology; SHUI Q. YE, MD, PhD, Director of Medical Genetics; MARA L. BECKER, MD, MSCE, Associate Professor of Pediatrics, Department of Pediatrics, Children's Mercy Hospital and Clinics, Kansas City, Missouri, USA. Address correspondence to Dr E. Fox, Children's Mercy Hospital, Department of Pediatrics, Graduate Medical Education, 2401 Gillham Road, Kansas City, MO 64108, USA. E-mail: ejfox@cmh.edu

Supported by grants from Katherine B. Richardson, Kansas City Area Life Sciences Institute, Children's Mercy Hospital Young Investigator Award, PhRMA Foundation Award, and the Paul Henson Award for Research in Immunology and Inflammation.

\section{ACKNOWLEDGMENT}

The authors thank Margaret Gibson and Li Zhang for helping with data collection, Drs. Andrew Lasky and Mark Hoeltzel for patient recruitment, and Chelsey Smith and Jaylene Weigel as research coordinators.

\section{REFERENCES}

1. Ye SQ, Simon BA, Maloney JP, Zambelli-Weiner A, Gao L, Grant A, et al. Pre-B-cell colony-enhancing factor as a potential novel biomarker in acute lung injury. Am J Respir Crit Care Med 2005; 171:361-70.

2. Fukuhara A, Matsuda M, Nishizawa M, Segawa K, Tanaka M, Kishimoto K, et al. Visfatin: A protein secreted by visceral fat that mimics the effects of insulin. Science 2005;307:426-30.

3. Revollo JR, Körner A, Mills KF, Satoh A, Wang T, Garten A, et al. Nampt/PFEB/visfatin regulates insulin secretion in $\beta$ cells as a systemic NAD biosynthetic enzyme. Cell Metab 2007;6:363-75.

4. Luk T, Malam Z, Marshall JC. Pre-B cell colony-enhancing factor (PBEF)/visfatin: A novel mediator of innate immunity. J Leukoc Biol 2008;83:804-16.

5. Rongvaux A, Shea RJ, Mulks MH, Gigot D, Urbain J, Leo O, et al. Pre-B-cell colony-enhancing factor, whose expression is up-regulated in activated lymphocytes, is a nicotinamide phosphoribosyltransferase, a cytosolic enzyme involved in NAD biosynthesis. Eur J Immunol 2002;32:3225-34.

6. Ozgen M, Suleyman SK, Aksoy K, Dagli N, Ustundag B, Isik A. Visfatin levels and intima-media thicknesses in rheumatic diseases. Clin Rheumatol 2011;30:757-63.

7. Neumann E, Frommer KW, Vasile M, Müller-Ladner U. Adipocytokines as driving forces in rheumatoid arthritis and related inflammatory diseases? Arthritis Rheum 2011;64:1159-69.

8. Brentano F, Schorr O, Ospelt C, Stanczyk J, Gay RE, Gay S, et al. Pre-B cell colony-enhancing factor/visfatin, a new marker of inflammation in rheumatoid arthritis with proinflammatory and matrix-degrading activities. Arthritis Rheum 2007;56:2829-39.

9. Moschen AR, Kaser A, Enrich B, Mosheimer B, Theurl M, Niederegger $\mathrm{H}$, et al. Visfatin, an adipocytokine with proinflammatory and immunomodulating properties. J Immunol 2007;178:1748-58.

10. Busso N, Karababa M, Nobile M, Rolaz A, Van Gool F, Galli M, et al. Pharmacological inhibition of nicotinamide phosphoribosyltransferase/visfatin enzymatic activity identifies a new inflammatory pathway linked to NAD. PLoS One 2008;3:e2267.

11. Beckers A, Organe S, Timmermans L, Vanderhoydonc F, Deboel L, Derua R, et al. Methotrexate enhances the antianabolic and antiproliferative effects of 5-aminoimidazole-4-carboxamide riboside. Mol Cancer Ther 2006;5:2211-17.

12. Fulco M, Cen Y, Zhao P, Hoffman EP, McBurney MW, Sauve AA, et al. Glucose restriction inhibits skeletal myoblast differentiation by activating SIRT1 through AMPK-mediated regulation of Nampt. Dev Cell 2008;14:661-73.

13. Katerelos M, Mudge SJ, Stapleton D, Auwardt RB, Fraser SA, Chen $\mathrm{CG}$, et al. 5-aminoimidazole-4-carboxamide ribonucleoside and AMP-activated protein kinase inhibit signaling through NF-KB. Immunol Cell Biol 2010;88:754-60.

14. Peiro C, Romacho T, Sánchez-Ferrer CF. Clinical implications of visfatin/PBEF/Nampt for development of vascular inflammation and atherosclerosis. JCMD 2011;2:34-8.

J Rheumatol 2013;40:5; doi:10.3899/jrheum.120639 